\title{
Measurement of residual strain in tantalum-clad tungsten after hot isostatic pressing
}

\author{
Dan Wilcox, Peter Loveridge, Saurabh Kabra, Tung Lik Lee, \\ Jeremy Moor, and David Jenkins
}

\section{Published version information}

Citation: D Wilcox et al. 'Measurement of residual strain in tantalum-clad tungsten after hot isostatic pressing.' Journal of Neutron Research, vo. 22, no. 2-3 (2020): 287-297.

DOI: $\underline{10.3233 / J N R-200181}$

The final publication is available at IOS Press through DOI above.

This version is made available in accordance with publisher policies. Please cite only the published version using the reference above. This is the citation assigned by the publisher at the time of issuing the AAM. Please check the publisher's website for any updates. 


\section{Measurement of residual strain in tantalum- 2 clad tungsten after hot isostatic pressing}

3 Dan Wilcox ${ }^{\mathrm{a}, *}$, Peter Loveridge ${ }^{\mathrm{a}}$, Saurabh Kabra ${ }^{\mathrm{a}}$, Tung Lik Lee ${ }^{\mathrm{a}}$, Jeremy Moor ${ }^{\mathrm{a}}$, and David

4 Jenkins $^{\mathrm{a}}$

$5 \quad{ }^{a}$ Rutherford Appleton Laboratory, Harwell Campus, Didcot, OX11 OQX, UK

Abstract. Tantalum-clad tungsten targets are a popular choice for spallation neutron production, due to the combination of high neutron yield and corrosion resistance. Such targets typically use the Hot Isostatic Press (HIP) process to bond the cladding to the core; this produces a strong bond but also introduces large residual stresses in the target and cladding. This is of particular interest at the ISIS neutron source, because cladding breaches are currently believed to limit the lifetime of ISIS TS2 targets. Two different and complementary methods were used to measure the residual strain in a tantalum-clad tungsten strip manufactured using the same HIP process as ISIS targets. The strip was produced with deliberately asymmetric cladding, causing it to deflect in proportion to the residual stress. FEA simulations were used to back-calculate the stress from the measured deflection. The strip was then placed on the ISIS instrument ENGIN-X, which allowed detailed through-thickness strain profiles to be measured via neutron diffraction. The results of both methods confirm the presence of large residual strains, and agree reasonably well with FEA simulations of the cladding process.

Keywords. Tantalum, Tungsten, Residual Stress, Hot Isostatic Press, Neutron Diffraction

\section{Introduction}

Tantalum-clad tungsten targets are a popular choice for spallation neutron production, due to the combination of high neutron yield and corrosion resistance. Such targets typically use the Hot Isostatic Press (HIP) method to bond the cladding to the core [1] [2]; this produces a strong diffusion bond between the materials, giving a good thermal connection, but simulations of the HIP process predict that it will also introduce large residual stresses in the target and cladding. The ISIS neutron source [3] currently operates two spallation targets, TS1 and TS2, both of which are tantalum-clad tungsten manufactured by HIPing. Any breach in the cladding will lead to radioactive tungsten corrosion products building up in the cooling water, which means the target must be replaced immediately. This is currently believed to be the lifetime limiting factor for ISIS TS2 targets. Understanding the residual stress state will help identify issues with current ISIS targets, and will be essential to developing a robust design for a future ISIS-II target that can withstand higher proton beam power. HIP-induced residual stress is currently an important but unknown factor in simulations of stress levels in ISIS targets. Finite Element Analysis (FEA) results indicate that residual stress, rather than beam-induced heating, is predicted to be the largest contribution to stress in tantalum for both TS1 and TS2 ISIS targets [4]. For simulation purposes, it is assumed that the residual stress is entirely due to differential thermal contraction between tantalum and tungsten as the part cools down after HIPing. This stress is assumed to be relieved at high temperatures, then start to build up below a certain 'lock-in' temperature. Estimating this lock-in temperature is an important step

*Corresponding author. E-mail: dan.wilcox@stfc.ac.uk. 
towards accurately simulating residual stress. More details of the standard ISIS HIP cycle and the methods and assumptions for simulating HIP stress can be found in [4].

Preliminary measurements of residual strain in a HIPed ISIS target plate have been carried out using neutron diffraction [5]. The results compared reasonably well with simulations of residual stress, as previously reported [4]. The experiment allowed the successful determination of two out of three strain components, but this was not enough to fully reconstruct the stress state in the material, so it was not possible to make an estimate of lock-in temperature. The two methods reported here aim to improve on this earlier method with an independent physical measurement, alongside an improved neutron diffraction method that builds on the experience gained from the earlier experiment.

\section{Asymmetrically-clad strip method}

\subsection{Sample manufacture}

A long, thin strip of tantalum-clad tungsten was manufactured by the ISIS target manufacturing group, using exactly the same processes and material specifications as the ISIS spallation targets. The cladding consists of several tantalum plates which were joined by electron beam welding under vacuum, then the assembly was HIPed with a peak temperature of $1200^{\circ} \mathrm{C}$ and a peak pressure of $140 \mathrm{MPa}$, which were held for 2 hours [4]. The strip was produced with deliberately asymmetric cladding on the upper and lower faces, causing it to deflect in proportion to the residual stress. The governing equations are similar to those for a bimetallic strip [6] with a third layer added, assuming edge effects are negligible. The dimensions of the strip were chosen such that the predicted deflection is large enough to be measured accurately, but the predicted stresses do not exceed the yield strengths of the materials. A parametric FEA study was carried out to determine the optimum dimensions, which were found to be as follows; a tungsten strip of 200x50x3mm, with $3.6 \mathrm{~mm}$ thick tantalum cladding on the sides and top face, and $1.6 \mathrm{~mm}$ thick tantalum on the bottom face. The completed strip is shown in Figure 1.

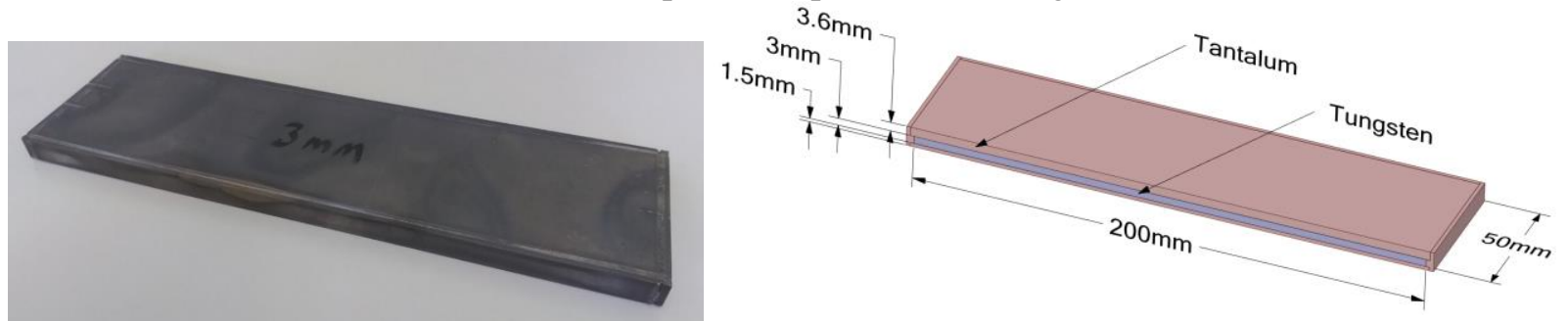

Fig. 1. Photograph of the manufactured asymmetrically-clad strip (left) and CAD model with key dimensions (right)

\subsection{Deflection results}

After HIPing, the deformed shape of the strip was measured using a Faro Edge arm-mounted laser scanner coordinate measuring machine. The machine's software compares measured points to a CAD model of the nominal component and returns a cloud of measured points in the same coordinate system as the original model. These data points were imported into MATLAB [7], as shown in Figure 2. The side and end walls were removed from the file, along with the outer $5 \mathrm{~mm}$ of tungsten, as simulations indicate that edge effects will be significant in this region. This left an upper and a lower surface of points; according to theory, each surface should fit a section of a sphere with a constant radius of curvature. The MATLAB curve fitting toolbox was used to fit each group of points with a spherical surface, where the radius and the coordinates of the sphere center could be varied freely. A plot of the two fits is shown in 
Figure 3. The coefficient of determination ' $\mathrm{r}$ ' ' was 0.990 for both surfaces, indicating a good fit to the measured data. The radius of curvature from the fits is $11.4 \mathrm{~m}$ on the top side and $10.4 \mathrm{~m}$ on the bottom side. The $\pm 95 \%$ confidence bounds for the fit radii of curvature were less than $\pm 0.2 \%$ of the reported values. The other fit parameters confirm that the center of the spherical fits is well aligned with the center of the strip, as expected.

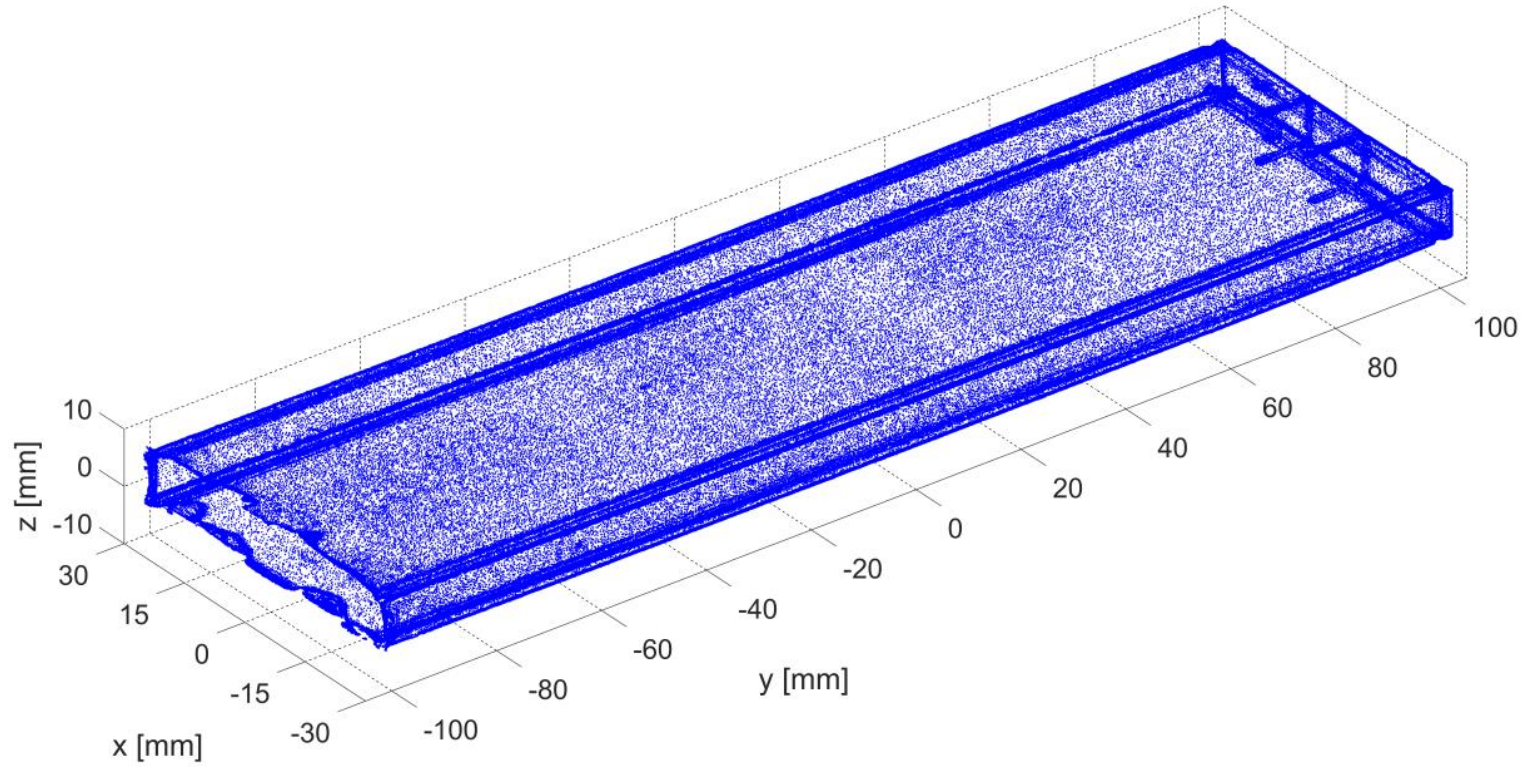

Fig. 2. Faro Edge coordinate measuring machine data points, after being imported into MATLAB. The missing face on the left of the picture is where the sample was supported during measurement.
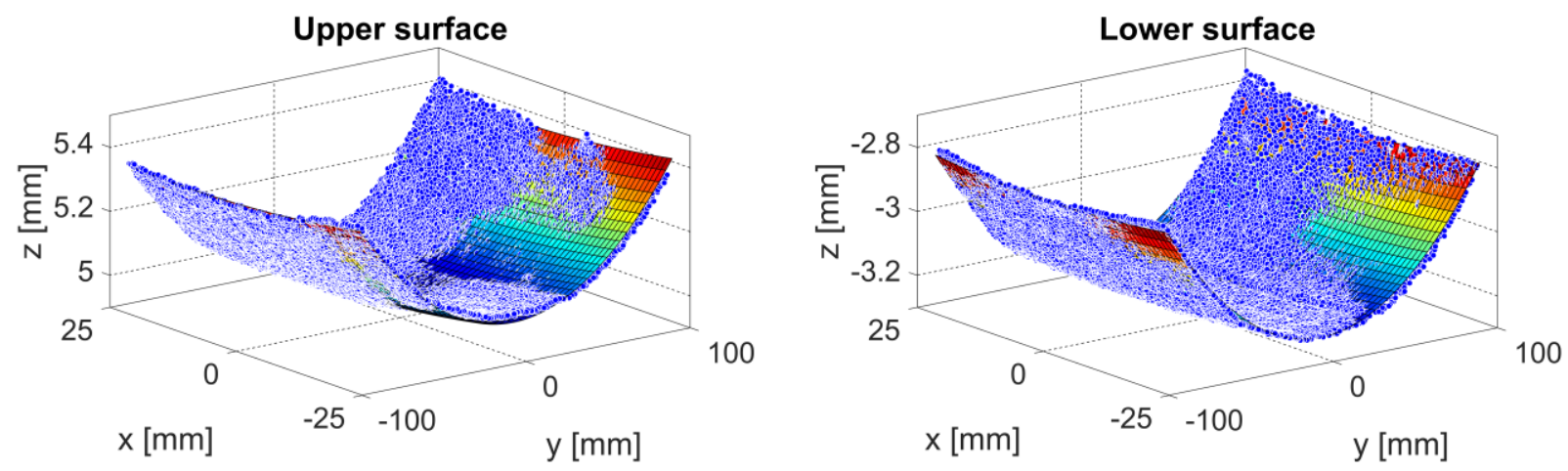

Fig. 3. Spherical MATLAB fits to the upper (left) and lower (right) surfaces of the asymmetrically-clad strip. Note that the axis scales are non-uniform in order to make the curvature and fit accuracy more clearly visible.

\subsection{Back-calculation of lock-in temperature}

The method for simulating residual stress in ISIS targets has previously been reported in detail elsewhere [4]. The same ANSYS simulation method was used to predict residual stress and deflection in the asymmetrically-clad strip. The simulated strip is assumed to be initially unstressed at the lock-in temperature, then is cooled down to $20^{\circ} \mathrm{C}$. The tantalum and tungsten are assumed to be perfectly bonded together, representing a successful HIP-induced diffusion bond. Temperature varying material properties 
were included for tantalum and tungsten. A bilinear kinematic hardening model was used to model yielding and plastic deformation of tantalum. A mesh independence study was carried out to ensure there were enough elements to accurately capture the through-thickness strain profile. There were two major unknowns in the simulation; lock-in temperature and tantalum yield strength. Previously conducted tensile tests on ISIS tantalum found yield strength values in the range 160-200MPa [8]. The lock-in temperature could in theory be anywhere between room temperature and the maximum HIP temperature of $1200^{\circ} \mathrm{C}$. Both of these parameters were varied parametrically in the ranges given.

A typical deformation plot is shown in Figure 4. The deformed upper surface fits a sphere of constant radius of curvature, as expected. Figure 5 shows how simulated radius of curvature varies as a function of lock-in temperature and tantalum yield strength ' $\sigma_{\mathrm{y}}{ }^{\mathrm{Ta}}$. The maximum and minimum measured radii of curvature from the MATLAB fits are also plotted. Based on the uncertainties in measured radius of curvature and yield strength, the lock-in temperature appears to be somewhere in the range 395 to $470^{\circ} \mathrm{C}$.

\section{A: Static Structural $200 \mathrm{~mm}$}

Directional Deformation

Type: Directional Deformation(Z Axis)

Unit: mm

Global Coordinate System

Time: 1

Fig. 4. ANSYS simulation of out-of-plane deformation ' $\mathrm{uz}_{\mathrm{z}}$ ' with a tantalum yield stress of $200 \mathrm{MPa}$, and a lock-in temperature of $500^{\circ} \mathrm{C}$. 


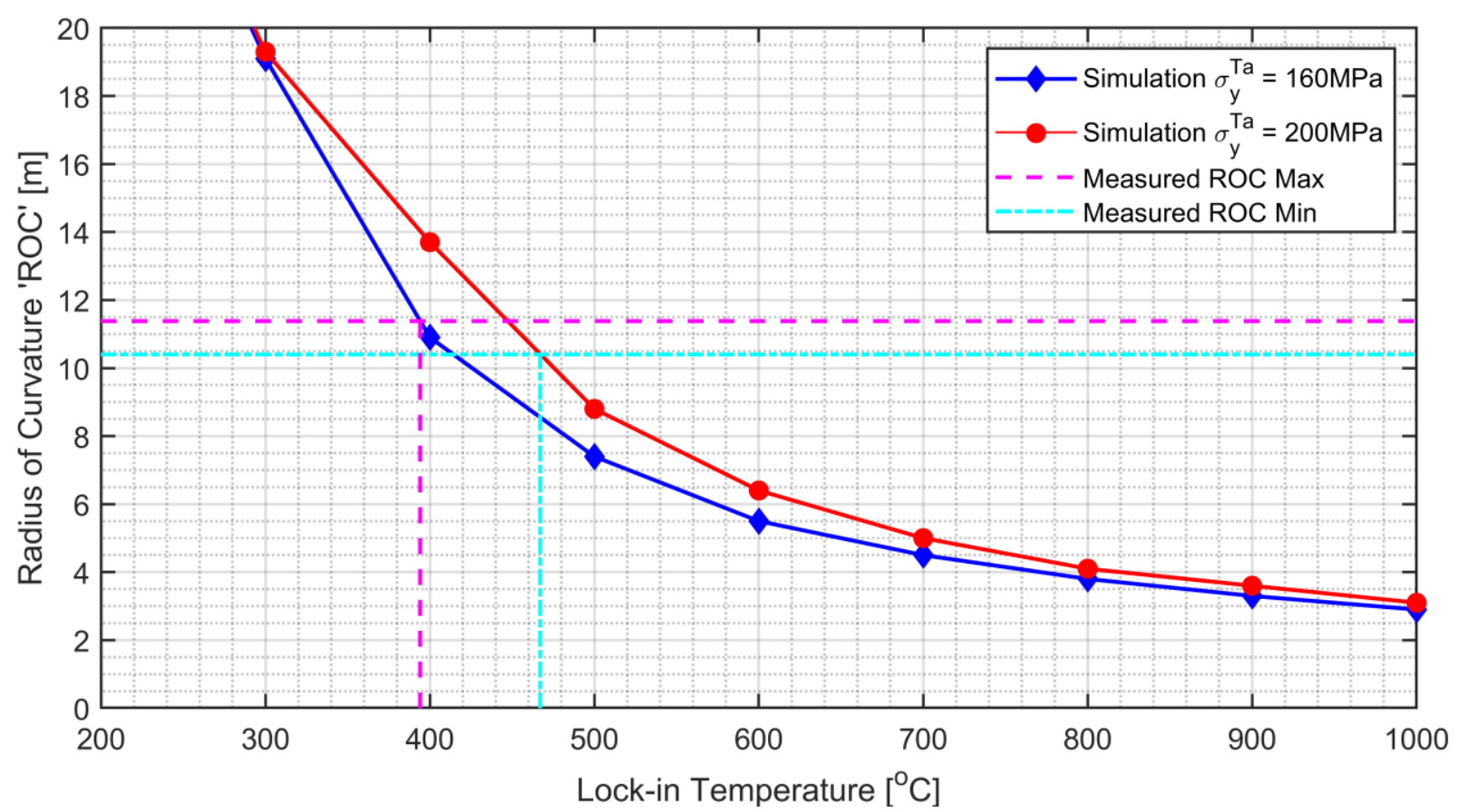

2 Fig. 5. Sensitivity of simulated radius of curvature (ROC) to lock-in temperature and tantalum yield strength $\left(\sigma_{\mathrm{y}}^{\mathrm{Ta}}\right)$.

\section{Neutron diffraction method}

\subsection{Experiment set-up}

The asymmetrically-clad strip was placed on the ISIS neutron diffraction instrument ENGIN-X [9] for two separate runs; the first for three days in October 2018 and the second for two days in March 2019. Through-thickness strain profiles were measured at various locations on the strip, as shown in Figure 6 . One location was measured in detail, with nine points on the through-thickness profile; three in the thick tantalum layer, three in tungsten, one in thin tantalum, and one at each of the two interfaces. Three additional locations were measured in less detail, with three points through-thickness; one each in the thick tantalum, tungsten, and thin tantalum. ENGIN-X measures two strain components at a time, so the sample had to be rotated part way through the experiment in order to measure all three strain components. This meant that the normal direction was measured twice, which was used as a cross check. Slits and collimators were used to limit the gauge volume to $1 \times 1 \times 18 \mathrm{~mm}$, giving $1 \times 1 \mathrm{~mm}$ resolution in the plane of measurement. The Bragg peaks in the neutron diffractograms were fitted using the full-pattern Pawley refinement method with GSAS to derive the lattice spacing ' $d$ '.

Neutron diffraction measurements require a nominally unstressed sample of each material to be measured for comparison, referred to as ' $\mathrm{d}_{0}$ ' samples. For accurate results the $\mathrm{d}_{0}$ samples should be as close as possible to the real materials in terms of chemical composition and processing route. It was not possible to obtain samples from exactly the same material batch as the asymmetrically-clad strip, but samples were obtained from the same supplier, with the same specification and processing route. The tungsten was forged, while the tantalum was rolled and annealed. Both materials were commercially pure. The $\mathrm{d}_{0}$ samples consisted of $80 \times 15 \times 1.6 \mathrm{~mm}$ plates. Because the tungsten was not annealed after forging, the tungsten $\mathrm{d}_{0}$ samples were cut into combs with $1.6 \times 1.6 \times 25 \mathrm{~mm}$ teeth to relieve any residual stress left 
over from forging. Two identical $\mathrm{d}_{0}$ samples were made for each material, one of which was put through the standard ISIS HIP cycle while the other was left as-received. This allowed the effect of HIPing on pure materials to be investigated separately from the effect of HIPing on the clad strip. Figure 7 shows the strip and one set of $d_{0}$ samples mounted on ENGIN-X. The strip was held in place using several grub screws rather than a flat clamp, in order to avoid flattening the initially curved shape of the strip.

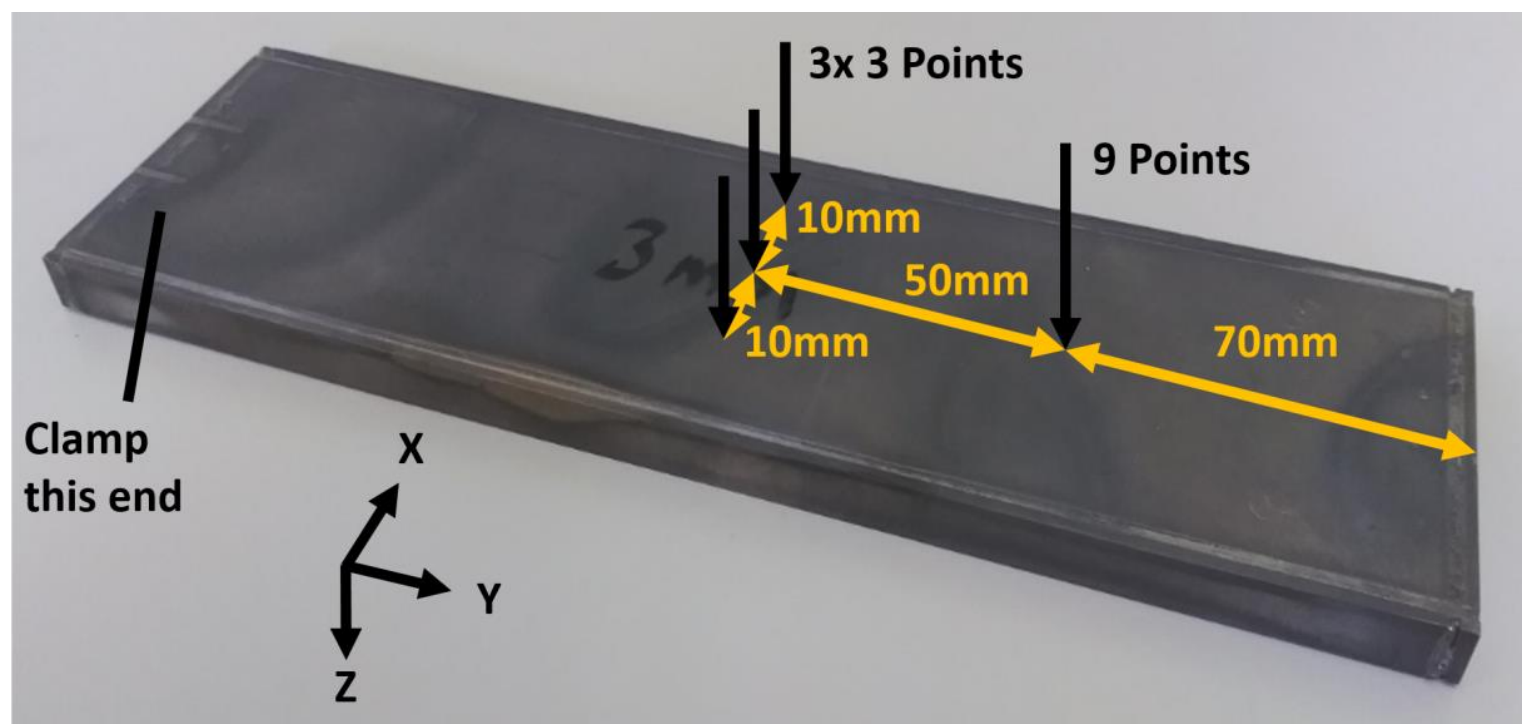

Fig. 6. Photograph of the manufactured asymmetrically-clad strip, with the location of the through-thickness neutron diffraction measurements, and the number of points in each measurement. The coordinate system shown is used for all presented results.
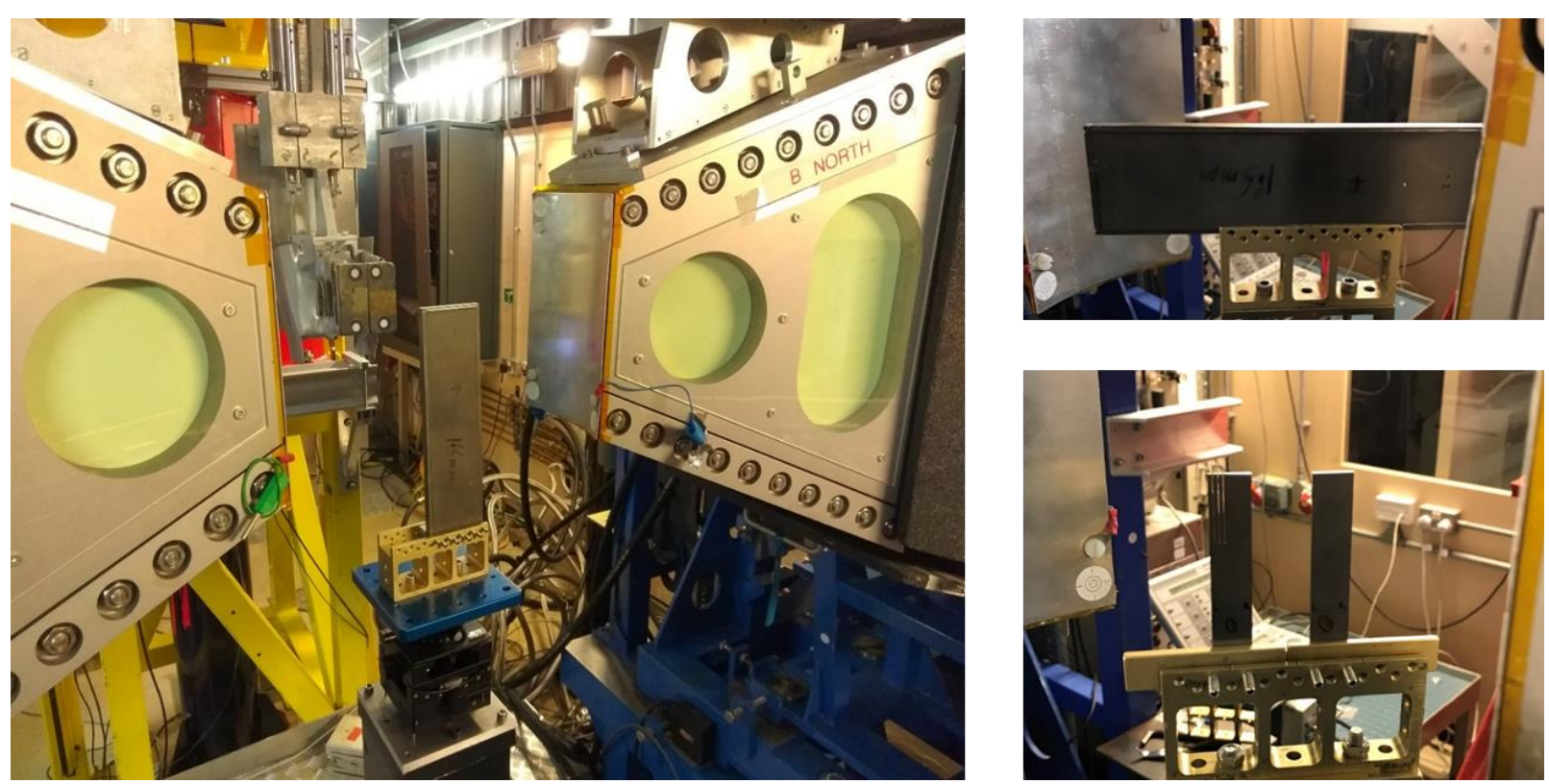

Fig. 7. The asymmetrically-clad strip mounted on the ENGIN-X instrument in the vertical (left) and horizontal (top right) orientations, and two do samples also mounted on ENGIN-X (bottom right). 
2 The measured through-thickness strain results are compared to the simulated strain profiles in Figure 3 8. The measured results are presented twice; using both the 'as received' and 'post HIP' $d_{0}$ samples. 4 Several ANSYS simulations were carried out, covering the expected range of uncertainties in the 5 simulation input parameters; lock-in temperature $\left(400-500^{\circ} \mathrm{C}\right)$ and tantalum yield strength $(160-200 \mathrm{MPa})$ 6 The interfaces between materials are clearly visible in both the simulations and the data. The interfaces 7 are at $\mathrm{Z}=3.6$ and $6.6 \mathrm{~mm}$. Repeated measurements were taken at $\mathrm{Z}=1.8,5.1$ and $7.4 \mathrm{~mm}-$ it can be seen 8 that the measurements agree very well between different locations. Because the strip was measured in 9 two different orientations, the strain in $\mathrm{z}$ was measured twice. The measurements agreed to within 100 10 microstrain, which helps to build confidence that the experiment has collected sufficient neutrons to yield 11 accurate statistics in the fitted data. Directions (tensile vs compressive) and magnitudes are generally as 12 expected - except for strain in the $\mathrm{z}$ direction in tantalum. This was expected to be highly compressive 13 due to the Poisson effect, as $\mathrm{x}$ and $\mathrm{y}$ directions are highly tensile, but it was measured to be zero or slightly 14 tensile.

15 Table 1 shows the strain in the HIPed $\mathrm{d}_{0}$ samples relative to the 'as-received' $\mathrm{d}_{0}$ samples. HIPing 16 induced a strain that was compressive in tungsten but tensile in tantalum, and the magnitude of which 17 was anisotropic. The uncertainty in the measurements is estimated to be around 100 microstrain, so the 18 change in the normal direction could be negligible, but there is definitely a significant effect in-plane.

19 Stress components were calculated from the measured strains using conventional solid mechanics. The 20 results are shown in Figure 9, and the Von Mises equivalent stresses are show in Figure 10. Note that 21 errors in the strain components could be multiplied when converting to stress.

22 Table 1. Strain in HIPed do samples, relative to 'as-received' $d_{0}$ samples. All values in microstrain.

\begin{tabular}{ccc}
\hline Material & In-Plane Strain & Normal Strain \\
\hline Tungsten & -294 & -58 \\
Tantalum & 309 & 83 \\
\hline
\end{tabular}



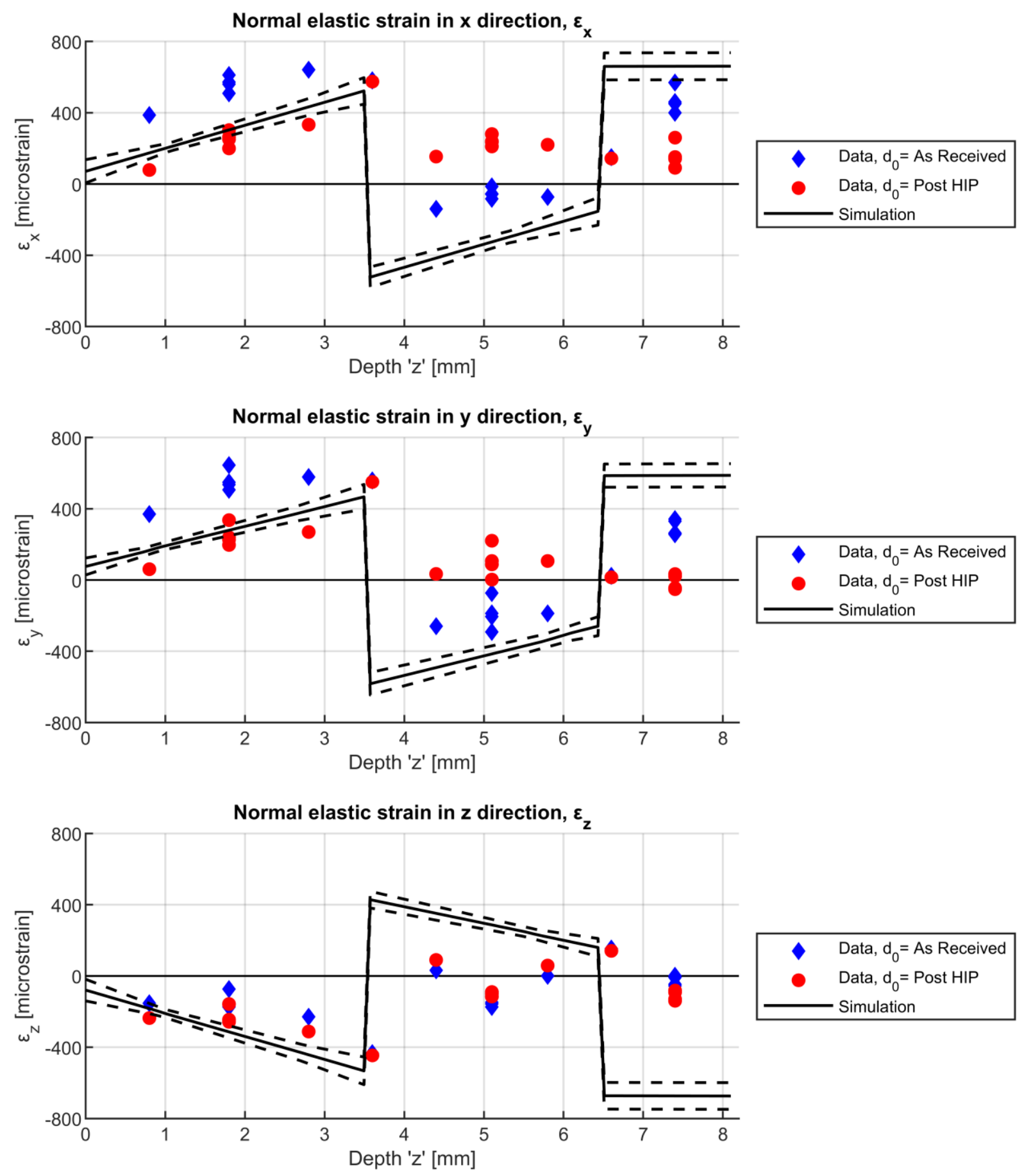

Fig. 8. Normal elastic strain values measured by neutron diffraction in the $\mathrm{x}, \mathrm{y}$ and $\mathrm{z}$ directions, compared to values from ANSYS simulation. The dotted lines represent the uncertainty in the simulation results due to uncertainties in the input values of lock-in temperature $\left(400-500^{\circ} \mathrm{C}\right)$ and tantalum yield strength $(160-200 \mathrm{MPa})$. 

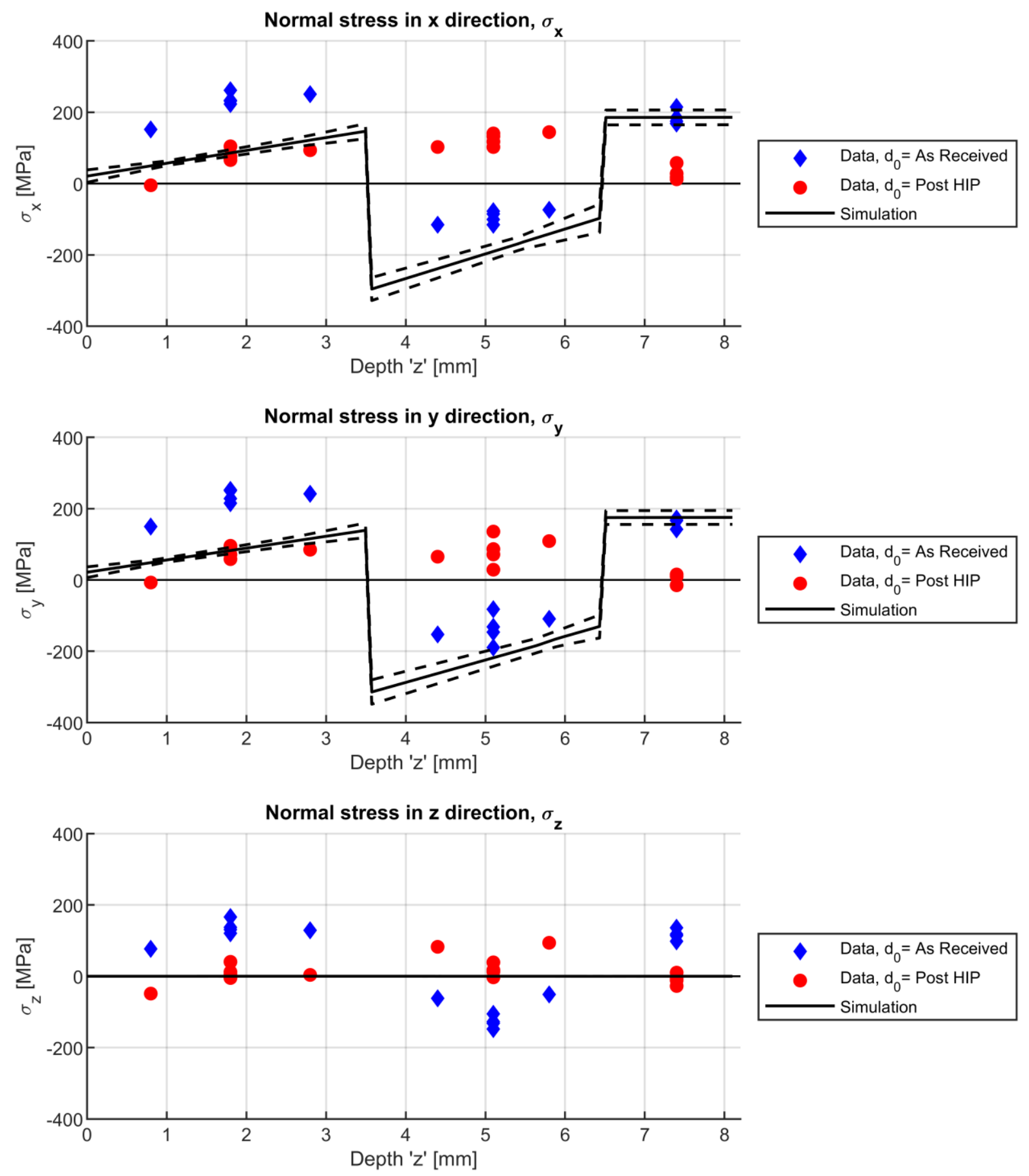

Fig. 9. Normal stress in the $\mathrm{x}, \mathrm{y}$ and $\mathrm{z}$ directions, as calculated from elastic strain values measured by neutron diffraction, compared to values from ANSYS simulation. The dotted lines represent the uncertainty in the simulation results due to uncertainties in the input values of lock-in temperature $\left(400-500^{\circ} \mathrm{C}\right)$ and tantalum yield strength $(160-200 \mathrm{MPa})$. Note that in the $\mathrm{z}$ direction, the ANSYS simulations predicted a maximum stress of less than $0.1 \mathrm{MPa}$, so the simulation line is nearly zero at all depths. 


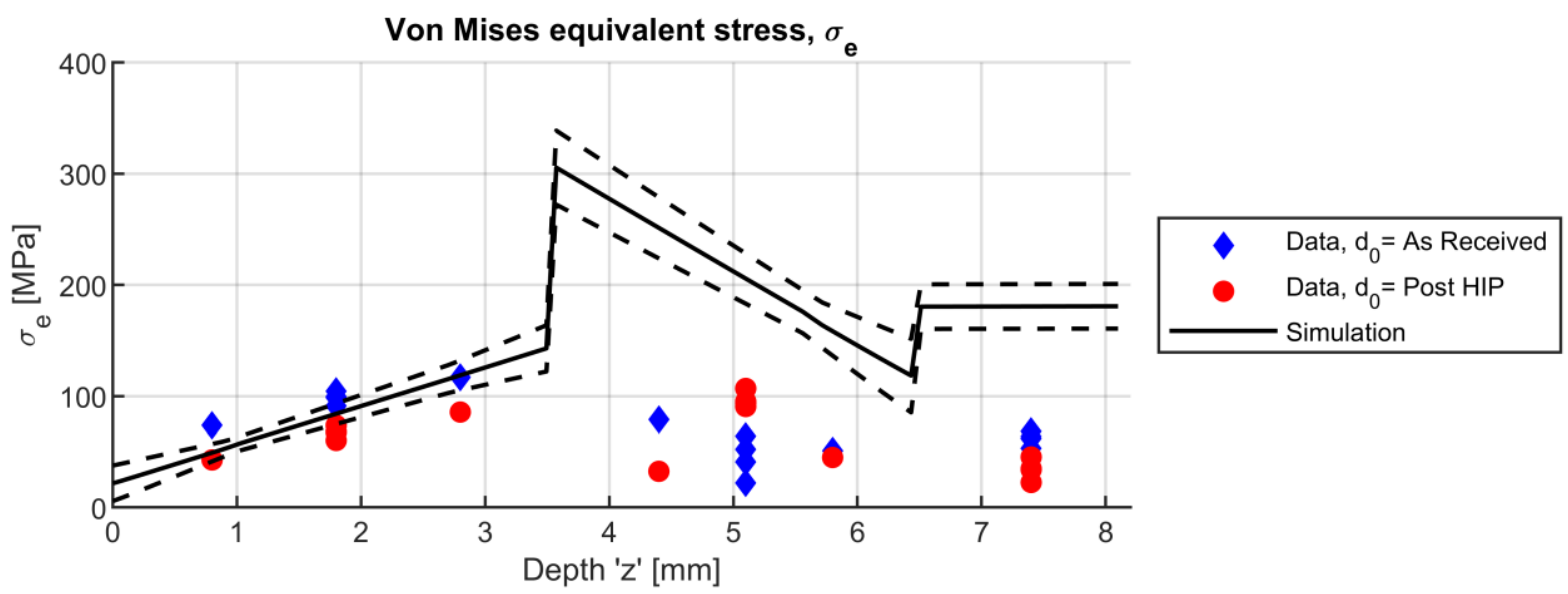

Fig. 10. Von Mises equivalent stress as calculated from elastic strain values measured by neutron diffraction, compared to values from ANSYS simulation. The dotted lines represent the uncertainty in the simulation results due to uncertainties in the input values of lock-in temperature $\left(400-500^{\circ} \mathrm{C}\right)$ and tantalum yield strength $(160-200 \mathrm{MPa})$.

\section{Discussion}

\subsection{Interpretation of experiments}

Both the physical measurement and the neutron diffraction results confirmed the presence of large residual stresses that are tensile in the cladding and compressive in the core, as expected. According to theory, the strain profile should be the same at all locations on the strip (excluding the edges), and have the same magnitude in both in-plane directions ( $\mathrm{x}$ and $\mathrm{y}$ ). The ENGIN-X measurements show fairly good agreement with these predictions, particularly if the 'as received' $\mathrm{d} 0$ measurement is used. Such a strain profile should produce a deflected surface that has a constant, uniform radius of curvature independent of position and orientation, i.e. the deflected surface should be a section of a sphere. Physical measurements of the strip confirmed that the deflected surfaces are indeed good fits to spherical surfaces.

Stress components were calculated from the ENGIN-X strain measurements. Like the strain profiles, the stress profiles agree fairly well with simulation in the $\mathrm{x}$ and $\mathrm{y}$ directions, particularly if the 'as received' $\mathrm{d} 0$ measurement is used, but do not agree well in the $\mathrm{z}$ direction. The simulation predicts negligible stress in the $\mathrm{z}$ direction, as the strip is thin in this dimension. Basic solid mechanics shows that the stress normal to an external surface must be zero. The measured stresses in the $\mathrm{z}$ direction do not meet these conditions, although using the 'post HIP' d0 measurement gives better agreement in this case. The Von Mises equivalent stress agrees well with the simulation in the thick tantalum layer, but does not agree well in the other two layers. The simulation predicts that the thin tantalum layer must have undergone some plastic deformation in order to produce the magnitude of deflection which was measured. Therefore the simulated Von Mises stress in the thin tantalum must be equal to the yield stress of tantalum; 160-200MPa. However, the Von Mises stress calculated from the ENGIN-X measurements is much lower. It is not possible to measure plastic strains using neutron diffraction, but the measured elastic strains do not appear to be consistent with plastic deformation having occurred in this region as expected. Note that errors in the strain measurements can be multiplied when converting strains to stresses. Uncertainties on stresses calculated from neutron diffraction will be particularly large in this case due to the high Young's moduli of tantalum and tungsten, which mean that large stresses only produce relatively small strains, and all the measurement uncertainties apply to the strains. 
Simulations with lock-in temperatures in the range 395 to $470^{\circ} \mathrm{C}$ predict the shape of the deformed strip with high accuracy, suggesting that the bulk deflections and stresses are being modelled fairly accurately. However there are details in the ENGIN-X results that are not well explained by the simulations. Various combinations of input parameters were tried, but none were able to accurately reproduce all of the measured data. Because of this, it was not possible to back-calculate an accurate estimate of lock-in temperature from this method. This suggests that there may be some real effects that are not accounted for in the simulations. In particular the effect of HIPing on the $d_{0}$ samples is not well understood; this is covered in more detail in section 4.2.

Two sets of ENGIN-X measurements were made, five months apart. Simple deflection measurements with a feeler gauge were made after the manufacture of the strip in 2017 and again two years later. Neither method found any evidence of stress relaxation over time. Once a real target is placed in a proton beam, there may be some stress relieving due to a combination of pulsed stresses and irradiation damage from the beam. However, the target becomes highly radioactive after only a short time in beam, so it would be very difficult to make any strain measurements on a post-irradiation target.

\subsection{Possible mechanisms not included in current simulations}

Neutron diffraction measurements of pure tantalum and tungsten $\mathrm{d}_{0}$ samples before and after HIPing revealed fairly large, anisotropic changes in measured strain, which was unexpected. In theory there should be no residual strain resulting from HIPing of a pure material. However there are some effects that could change the measured lattice spacing, such as picking up impurities from the HIP atmosphere. This would be expected to change the measured strain uniformly in all directions, which is not what was observed. This anisotropy makes it particularly difficult to apply a correction factor to other geometries such as the strip.

It is possible that the strip also contains some residual strains from the initial welding operations, or from deformation under pressure during the HIP process. These are expected to be completely relieved due to the high HIP temperature, and are therefore not included in the FEA simulation. If some of these strains remained after HIPing, this could affect the measured strain profiles. However, strains due to welding would be concentrated at the plate edges, and the magnitude of pressure-induced strains would vary with location. As the measured strain profiles did not vary significantly with position on the plate surface, these effects do not explain the observed differences between simulation and measurement. Welding is also known to increase the tantalum grain size in the heat affected zone, but the strain measurements were made well away from this zone.

Both the tantalum and the tungsten may have a textured microstructure as a result of rolling/forging. This could lead to anisotropic properties in the bulk material that were not taken into account during the simulations (in which the material was assumed to be an isotropic continuum). The ISIS HIP recipe deliberately uses a HIP temperature lower than the recrystallisation temperature in order to prevent grain growth. However if the HIP does change the texture of the microstructure, this could explain the anisotropic changes in $\mathrm{d}_{0}$ measurement after HIPing samples of pure tantalum and tungsten.

Previous measurements of tensile properties of HIPed and unHIPed ISIS tantalum show that HIPing can have a fairly large effect on Young's modulus [8]. This effect is not accounted for in the simulations, and no measurements have been made of whether or not the change in Young's modulus is isotropic. The cause of this effect is not known, but it could indicate a change in impurities or microstructure. Further investigation of HIP effects on the microstructure and material properties is recommended, perhaps via microscopy and hardness testing of the HIPed and unHIPed $\mathrm{d}_{0}$ samples.

There could also be some creep occurring in the tantalum, either during the cooldown after HIPing, or room temperature creep after the process has finished. ISIS tantalum has previously been found to exhibit what appears to be room temperature creep, starting at a stress somewhere between 100 and 150MPa [10]. 
The simulated stress in the asymmetrically-clad strip exceeds $100 \mathrm{MPa}$ for most assumed lock-in temperatures, so this is a possibility. Creep strain would be essentially plastic, and therefore would not appear in neutron diffraction measurements. ANSYS has various options for simulating creep, but the necessary material properties for tantalum are currently not known. A program of physical testing would be required in order to measure the creep rate as a function of temperature and stress, then an appropriate creep law could be chosen to fit the data.

\subsection{Implications for target design}

The presence of large residual stresses from HIPing has been confirmed, so this should be taken into account in future target analysis work. A significant effect of this residual stress is thought to be a reduction in the fatigue life of the cladding due to the high tensile stress. Simulations of current ISIS targets show a large safety factor on fatigue due to combined residual stress and operational loads. However, residual stress could reduce the operational lifetimes of higher power targets in a potential future ISIS-II development. There is currently very limited data on combined radiation damage and fatigue effects in tantalum. If radiation damage causes the tantalum to lose all ductility then the high tensile residual stress could become a problem. The residual stress profile may also affect the propagation and intensity of dynamic stress waves following each beam pulse. The trend in modern spallation facilities is towards ever higher beam powers, and therefore higher stresses in targets. There is also a desire to reduce cladding thicknesses in order to minimise decay heat production, which is often dominated by the presence of tantalum-182. Developing a better understanding of residual stresses and other effects that could compromise cladding integrity is therefore increasingly important to ensure target integrity.

\section{Conclusion}

Two different and complementary methods were used to measure the residual strain in a tantalum-clad tungsten strip manufactured using the same HIP process as ISIS targets. The results of both methods confirm the presence of large residual strains, and agree reasonably well with FEA simulations of the cladding process. Comparison of simulated and measured deflection results indicates a lock-in temperature of between 395 and $470^{\circ} \mathrm{C}$. The neutron diffraction results suggest that there may be some additional effects that are not included in the simulation. In particular, HIPing of pure tantalum and tungsten produces unexpected changes in strain measurement. It is currently not clear if this is a change in strain, or a change in the measured lattice spacing. Further investigation of the effects of HIPing on impurities and microstructure is recommended. Continuing to develop a better understanding of stress levels and failure modes will enable more optimised targets and higher beam powers in future.

\section{Acknowledgements}

We thank the ISIS neutron facility for providing beamtime on the ENGIN-X beamline, experiment DOI 10.5286/ISIS.E.RB1830588. Manufacture of the asymmetrically-clad strip was carried out by Jeremy Moor, Max Rowland and Peter Webb of the ISIS target manufacturing group. Engineering drawings were produced by Leslie Jones of the ISIS target design group. Many thanks to Phil Earp of the UK Atomic Energy Authority for assistance with proof-reading and corrections. 


\section{References}

[1] M. Kawai et al., "Fabrication of a tantalum-clad tungsten target for KENS," Journal of Nuclear Materials, no. 296, pp. 312320, 2001. A.N. Author, Article title, Journal Title 66 (2005), 856-890. doi:10.1234/JN-2005-102-203.

[2] A. T. Nelson, J. A. O'Toole, R. A. Valicenti and S. A. Maloy, "Fabrication of a tantalum-clad tungsten target for LANSCE," Journal of Nuclear Materials, vol. 431, p. 172-184, 2012.

[3] D. J. S. Findlay, "ISIS - Pulsed Neutron and Muon Source," in Proceedings of PAC07, Albuquerque, 2007.

[4] D. Wilcox, P. Loveridge, T. Davenne, L. Jones and D. Jenkins, "Stress levels and failure modes of tantalum-clad tungsten targets at ISIS," Journal of Nuclear Materials, vol. 506, pp. 76-82, 2018.

[5] Yanling Ma, et al., "An Experiment Using Neutron Diffraction to Investigate Residual Strain Distribution in a Hot Isostatic Pressed (HIPPED) Target Plate," in Joint 3rd UK-China Steel Research Forum \& 15th CMA-UK Conference on Materials Science and Engineering, 2014.

[6] S. Timoshenko, "Analysis of Bi-Metal Thermostats," Journal of the Optical Society of America, vol. 11, no. 3, pp. 233-255, 1925.

[7] "MATLAB R2018b", The MathWorks, Inc.

[8] E. Quinn, "Material Properties Evaluation of a Hot Isostatically Pressed Tantalum Pressure Vessel for the ISIS TS-2 Target," 2012. [Online]. Available: http://pasi.org.uk/images_pasi/3/31/IWSWT11_Ta_HIP.pdf. [Accessed 18 December 2019].

[9] J. R. Santisteban et al., "ENGIN-X: a third-generation neutron strain scanner," Journal of Applied Crystallography, no. 39, p. 812-825, 2006.

[10] C. Nelson, "Material Properties of Tantalum including High Cycle Fatigue," Proc. 14th Int. Workshop Spallation Materials Technology, JPS Conf. Proc. 28, 081005, 2020. 